Egyptian

Orthodontic Journal

\title{
EVALUATION OF CROWN-ROOT ANGULATION OF LATERAL INCISOR ADJACENT TO IMPACTED CANINES USING PANORAMA AND CBCT
}

\author{
Nahla E Gomaa MD ${ }^{(1)}$, Mohammed M Ellaithy MD(1)
}

\section{ABSTRACT:}

Maxillary canines are the most commonly impacted teeth, second only to third molars. Maxillary canine impaction occurs in approximately $2 \%$ of the population and is twice as common in females as in males. Approximately one-third of impacted maxillary canines are located labially and two-thirds are located palatally. Lateral incisor demonstrates an important role in the etiology of palatally impacted canines. So, the objective of this study is to compare the difference in the shape of lateral incisor (crown-to-root angulation) adjacent to palatally and labially impacted canine using conventional 2D and $3 \mathcal{D}$ radiographs. Sample of this study consisted of 60 patients (32 females and 28 males) with a mean age of 13.94 years. For each subject, two sets of radiographs (panoramic and CBCT scan) had been obtained. These patients were divided into 2 groups: The first group consisted of 35 patients with palatally impacted maxillary canines; the second group consisted of 25 patients with labially impacted maxillary canines. The control group (third group) consisted of normally erupted maxillary canines (25 subjects). Crown to root angulations of lateral incisors were measured. $t$ test for independent samples was used to assess the crown-root angulation of lateral incisors. The results revealed that There was

1- Lecturers of Orthodontics, Orthodontic department, Faculty of Dentistry, Tanta University, Egypt. 
Egyptian

Orthodontic Journal

no significant difference for crown root angulation of Cateral incisor between palatally and labially impacted canines groups using panoramic $x$-ray or $C B C T(P=0.831,0.395$ respectively). Insignificant difference was found between panorama and CBCT in determining crown-root angulation of lateral incisor. In impacted canines groups, the roots of the adjacent lateral incisors were more angulated compared to those adjacent to normally erupted canines.

\section{INTRODUCTION}

Canines are the cornerstone of dental arches. Maxillary canines contribute fundamentally to the stylish and biting capacities, any unsettling influences in the emission or eruption of permanent maxillary canines can bring about problems in the dental arch and contiguous teeth, which require special consideration ${ }^{[1]}$.

Impacted canines are a frequently encountered problem in orthodontics $^{[2]}$. Impacted canines are defined as a state where a tooth stays inserted in the oral mucosa or bone past its normal period. This definition can be expanded to incorporate teeth that are anticipated to experience abnormal eruption, even before its normal eruption period, due to position of tooth germ, tooth shape, direction of eruption and accessible space ${ }^{[3]}$.

The maxillary canines are the most frequently impacted teeth after the third molars with a prevalence rate ranging between 1-3\%. Canines may be impacted palatally or labially ${ }^{[4]}$. Maxillary canines impactions cause aesthetic problems, like tipping of adjacent teeth, midline deviation and root resorption on adjacent teeth ${ }^{[3]}$.

Genetic and guidance theories are the primary theories that can clarify the development of palatally impacted canines. Genetic theory proposes that impaction is because of genetic predisposition; bolstered by proof uncovering a relationship between palatally impacted canines and 
Egyptian

Orthodontic Journal

other dental varieties of genetic origin, for example, agenesis of lateral incisors, small lateral incisor crown size, distal displacement of mandibular second premolars, aplasia of premolars and third molars, and tooth transposition ${ }^{[4]}$.

The maxillary canines are prone to be deviated from the normal path of eruption as their total eruption path extends over $2 \mathrm{~cm}$ between the age of $5-15$ years $^{[5]}$.

Ordinary two dimensional (2D) radiographic imaging was the most widely recognized methodology used to restrict impacted canines, treatment arranging and assessment of treatment results. All-encompassing radiograph is considered as a standard symptomatic guide in orthodontics utilized for preoperative determination of routine cases. In any case, the precision and validity for confinement of impacted canines and nearby structures might be disparaged because of lacks, for example, distortion projection blunders, blurred images and complex maxillofacial structures that are anticipated onto a 2D plane prompting misinterpretation.

As of late, cone beam computed (CBCT) unit has been presented with lessened radiation exposure and 3D imaging capacity for dental structures $^{[6]}$.

As said some time recently, the exact etiology of palatally impacted canines is obscure, it gives the thought that neighboring lateral incisor assume a basic part, either in light of the way that its eruption and measurements are controlled by the same genes that control the eruption or emission of the canine (genetic hypothesis), or in light of the fact that its position in the arch impacts the eruption path of the canine (guidance theory) ${ }^{[4]}$. So, the aim of this study was to compare the difference in the shape of lateral incisor (crown-toroot angulation) adjacent to palatally and labially impacted canine using conventional 2D and 3D (CBCT) radiographs.

\section{METHODS}

Sample of this study was patients in the Orthodontic Department, Faculty of Dentistry, Tanta University. The research sample consisted of 60 patients (32 females and 28 males). The age of the patients was

Volume 49 - June 2016 
between 12 and 17.5 years of age, with a mean age of 13.94 years. For each subject, two sets of radiographic information had been obtained. First set consisted of traditional panoramic radiographs and the second set consisted of 3D volumetric images obtained from CBCT scan (Planmeca, proline xc, Helsinki, Finland). The scanning conditions were $90 \mathrm{kV}$, $4 \mathrm{~mA}$. field of view $20 \times 19 \mathrm{~cm}$, scan time: 24 seconds, voxel size: $0.4 \mathrm{~mm}$.

These patients were divided into 2 groups: The first group consisted of 35 patients with palatally impacted maxillary canines, the second group consisted of 25 patients with labially impacted maxillary canines (The impacted canines have no sign of eruption in the oral cavity).The control group (third group) consisted of normally erupted maxillary canines (25 subjects), selected from the archives of the orthodontic clinic. The aim of this study was to compare the difference in the shape of lateral incisor (crownto-root angulation) adjacent to palatally and labially impacted maxillary canines. So, each maxillary canine was considered as an individual sample. Meaning that, if a subject had two labially impacted canines, so we had two study samples. Also, in palatally impacted canines, there were two samples. But, in subjects with unilateral impaction, we had only one study sample, because the morphology of the lateral incisor is affected by genetic predisposition so, the lateral incisor on the contralateral side was not considered as a control. Unclear root morphology of lateral incisor was excluded. To have a better representation of the direction of long axis of lateral incisor root, dilacerations at its root apex was not considered. Cone beam technique was used to differentiate between palatally and labially impacted canines.

A positive degree measurement was recorded when long axis of the root was angulated mesially in relation to that of the crown, and a negative degree measurement was recorded when long axis of the root was angulated distally.

Student's $\mathrm{t}$ test for independent samples was used to assess the crown-root angulation of lateral incisors. $\mathrm{P}$ value less than 0.05 was considered significant. 
Egyptian

Orthodontic Journal

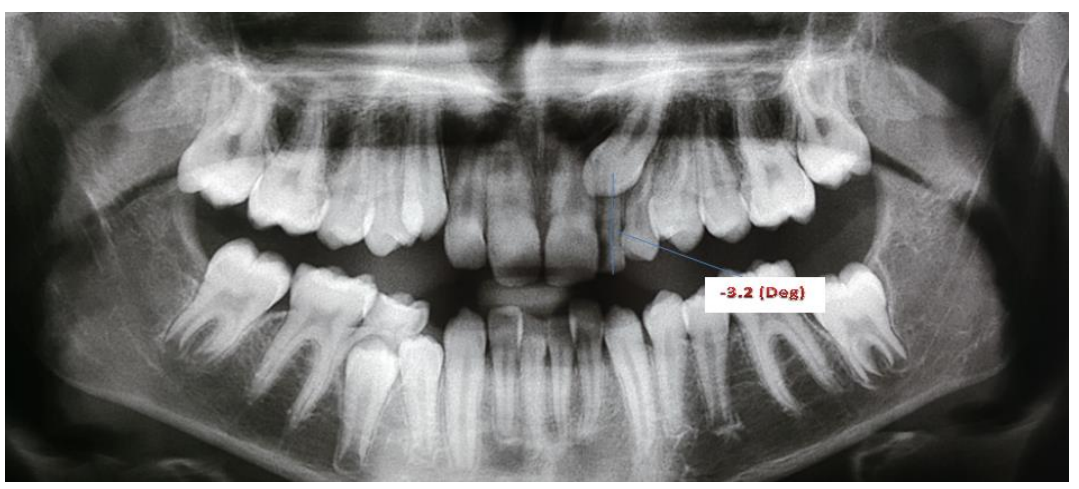

(Figure 1) Crown-root angulation of maxillary lateral incisor using panoramic radiograph.
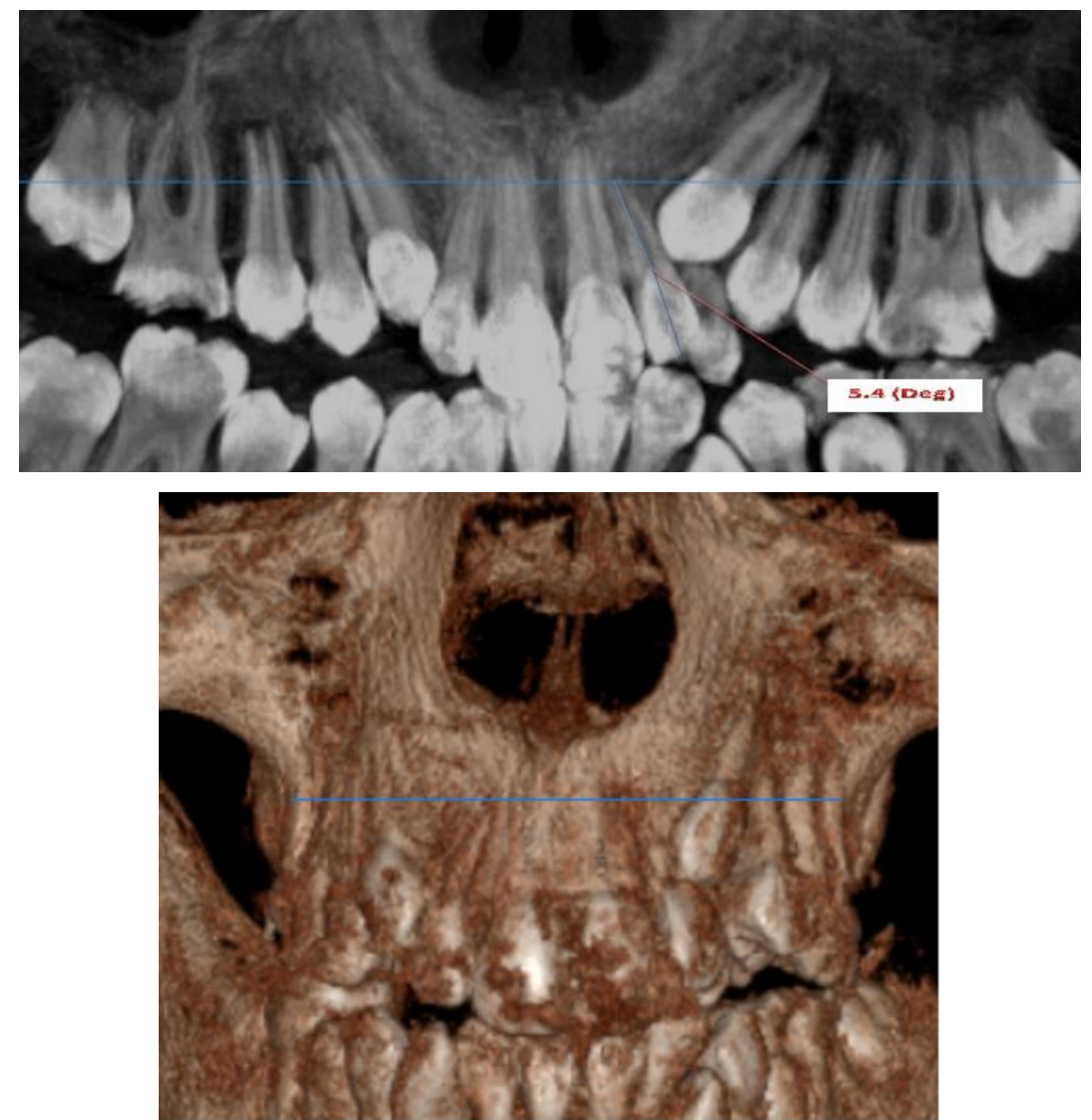

(Figure 2) Crown-root angulation of maxillary lateral incisor using CBCT. 
Egyptian

Orthodontic Journal

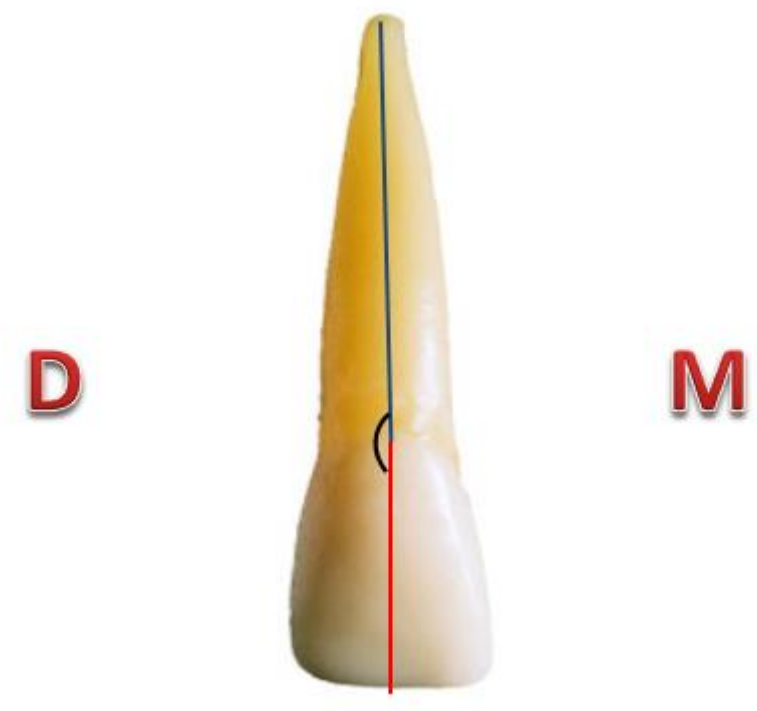

(Figure 3) Diagram of crown-root angulation of maxillary lateral incisor.

\section{RESULTS}

Mean and standard deviation for crown root angulation of lateral incisor of palatally and labially impacted canines using panoramic $\mathrm{x}$-ray are shown in Table 1.

There was no significant difference between the two groups $(\mathrm{P}=0.831)$.

Also, results revealed an insignificant difference between palatally and labially impacted canines in CBCT group $(\mathrm{P}=0.395)$. Table 2 .

No significant difference was found between panorama and CBCT in crown root angulation in both groups $(\mathrm{P}=0.850$ and $\mathrm{P}=0.292$ respectively) Table 3, 4 .

As regard control group, the outcome of this study showed that mean value of crown root angulation of lateral incisor adjacent to palatally or labially impacted canines (which is considered abnormal) is higher than that adjacent to normally erupted canines even in panoramic or CBCT. Table 5, 6 . 
Egyptian

Orthodontic Journal

Table 1: Range, mean and standard deviation for crown root angulation of lateral incisor of palatally and labially impacted canines using panoramic x-ray

\begin{tabular}{|c|c|c|c|c|c|c|}
\hline & \multicolumn{2}{|c|}{ Panorama } & \multicolumn{2}{|c|}{ Difference } & \multicolumn{2}{|c|}{ Paired T-test } \\
\hline & 1st group & 2nd group & Mean & SD & $\mathbf{T}$ & P-value \\
\hline Range & $0: 8$ & $-6: 8$ & \multirow{2}{*}{0.286} & \multirow{2}{*}{4.906} & \multirow{2}{*}{0.218} & \multirow{2}{*}{0.831} \\
\hline Mean \pm SD & $4.714 \pm 2.335$ & $4.429 \pm 4.783$ & & & & \\
\hline
\end{tabular}

Table 2: Range, mean and standard deviation for crown root angulation of lateral incisor of palatally and labially impacted canines using CBCT:

\begin{tabular}{|l|c|c|c|c|c|c||}
\hline \multirow{2}{*}{} & \multicolumn{2}{|c|}{ CBCT } & \multicolumn{2}{c|}{ Difference } & \multicolumn{2}{c|}{ Paired T-test } \\
\cline { 2 - 7 } & 1st group & 2nd group & Mean & SD & T & P-value \\
\hline Range & $-5.1: 9$ & $-2.2: 9.5$ & & & & \\
\cline { 1 - 3 } Mean \pm SD & $4.986 \pm 4.555$ & $6.121 \pm 3.678$ & & & & \\
\hline
\end{tabular}

Table 3: Range mean and standard deviation of crown root angulation in palatally impacted canines group (1st group) using panorama and CBCT:

\begin{tabular}{|c|c|c|c|c|c|c|}
\hline & \multicolumn{2}{|c|}{ 1st group } & \multicolumn{2}{|c|}{ Difference } & \multicolumn{2}{|c|}{ Paired T-test } \\
\hline & Panorama & CBCT & Mean & SD & $\mathbf{T}$ & P-value \\
\hline Range & $0: 8$ & $-5.1: 9$ & \multirow{2}{*}{-0.271} & \multirow{2}{*}{5.256} & \multirow{2}{*}{-0.193} & \multirow{2}{*}{0.850} \\
\hline Mean \pm SD & $4.714 \pm 2.335$ & $4.986 \pm 4.555$ & & & & \\
\hline
\end{tabular}

Table 4: Range mean and standard deviation of crown root angulation in labially impacted canines group (2nd group) using panorama and CBCT:

\begin{tabular}{|c|c|c|c|c|c|c|}
\hline & \multicolumn{2}{|c|}{ 2nd group } & \multicolumn{2}{|c|}{ Difference } & \multicolumn{2}{|c|}{ Paired T-test } \\
\hline & Panorama & CBCT & Mean & SD & $\mathbf{T}$ & P-value \\
\hline Range & $-6: 8$ & $-2.2: 9.5$ & \multirow{2}{*}{-1.693} & \multirow{2}{*}{5.768} & \multirow{2}{*}{-1.098} & \multirow{2}{*}{0.292} \\
\hline Mean \pm SD & $4.429 \pm 4.783$ & $6.121 \pm 3.678$ & & & & \\
\hline
\end{tabular}

Volume 49 - June 2016 
Egyptian

Orthodontic Journal

Table 5: Range, mean and standard deviation for crown root angulation of lateral incisor of control group, palatally and labially impacted canines using panoramic X-ray:

\begin{tabular}{|c|c|c|c|c|c|}
\hline & & \multicolumn{2}{|c|}{ Panorama } & \multicolumn{2}{|c|}{ T-test } \\
\hline & & Panorama & Controls & $\mathbf{T}$ & P-value \\
\hline \multirow{2}{*}{ 1st group } & Range & $0: 8$ & $-1: 0.5$ & \multirow{2}{*}{7.985} & \multirow{2}{*}{0.000} \\
\hline & Mean \pm SD & $4.714 \pm 2.335$ & $-0.393 \pm 0.525$ & & \\
\hline \multirow{2}{*}{ 2nd group } & Range & $-6: 8$ & $-1: 0.5$ & \multirow{2}{*}{3.749} & \multirow{2}{*}{0.001} \\
\hline & Mean \pm SD & $4.429 \pm 4.783$ & $-0.393 \pm 0.525$ & & \\
\hline
\end{tabular}

Table 6: Range, mean and standard deviation for crown root angulation of lateral incisor of control group, palatally and labially impacted canines using CBCT:

\begin{tabular}{||c|l|c|c|c|c||}
\hline \multirow{2}{*}{} & \multicolumn{2}{c|}{ CBCT } & \multicolumn{2}{c||}{ T-test } \\
\cline { 3 - 5 } \multicolumn{2}{||}{} & CBCT & Controls & T & \multirow{2}{*}{ P-value } \\
\hline \multirow{3}{*}{ 1st group } & Range & $-5.1: 9$ & $-1: 0.5$ & \multirow{2}{*}{4.389} & \multirow{2}{*}{0.000} \\
\cline { 2 - 4 } & Mean \pm SD & $4.986 \pm 4.555$ & $-0.393 \pm 0.525$ & & \\
\hline \multirow{2}{*}{ 2nd group } & Range & $-2.2: 9.5$ & $-1: 0.5$ & \multirow{2}{*}{6.560} & 0.000 \\
\cline { 2 - 4 } & Mean \pm SD & $6.121 \pm 3.678$ & $-0.393 \pm 0.525$ & & \\
\hline
\end{tabular}

\section{DISCUSSION}

For every orthodontist, treatment of impacted maxillary canines has been an interesting challenge from diagnostic and therapeutic point of view.

Abnormally shaped, peg or missing lateral incisors tend to guide the adjacent canine into an incorrect position in the dental arch. This concept is supported by some investigators. Others were suggesting that palatally impacted canines and abnormal shape of maxillary lateral incisor tend to occur concomitantly. 
Egyptian

Orthodontic Journal

So, the aim of this study was to compare the difference in crown-toroot angulation of lateral incisor adjacent to palatally and labially impacted canines using conventional 2D and 3D (CBCT) radiographs.

The sample of this research consisted of 60 patients. For each subject, two sets of radiographic information had been obtained. First set consisted of traditional panoramic radiographs and the second set consisted of 3D volumetric images obtained from CBCT scan. These patients were divided into 2 groups. The first group consisted of palatally impacted maxillary canines; the second group consisted of labially impacted maxillary canines. Control group (third group) composed of 25 subjects with normally erupted canines.

As the morphology of the lateral incisor is affected by genetic predisposition, the lateral incisor on the contralateral side of unilateral impacted canine was not considered as a control. So, subjects of control group were selected from the archives of the orthodontic clinic with no canine impactions.

Results of this study found that there was no significant difference in crown root angulation between palatally and labially impacted canine groups. A significant difference was present between these two groups and the control group. No significant difference was found between panorama and CBCT.

A significant difference was found in crown to root angulation between impacted canine (palatally and labially) groups and the control group.

The relation between palatally displaced canine and upper lateral incisor morphology was studied previously ${ }^{(7,8,9)}$. Becker et $\mathrm{al}^{(10)}$, Jacoby $^{(11)}$, Becker et $\mathrm{al}^{(12)}$, Peck et $\mathrm{al}^{(13,14)}$, Sacerdoti and Baccetti ${ }^{(9)}$, and Mossey et $\mathrm{al}^{(15)}$, found that presence of excess space in the maxillary arch(due to agenesis or shape anomaly of maxillary lateral incisor) can be a causative factor for the canine to deflect from its normal path of eruption. So, it can move more downward and directed palatally. They added that there is a relation or a connection between the gene or genes responsible for agenesis or hypodontia of the incisor and that gene or 
Egyptian

Orthodontic Journal

genes responsible for eruption or displacement of the canine. Liuk et al ${ }^{(16)}$ used cases with palatally impacted canines to compare the dimensions of lateral incisors to normal controls using cone-beam-computedtomography. They found that the lateral incisors had significantly smaller crown and root dimensions. Peck et al ${ }^{(8)}$ and Sacerdoti and Baccetti (9) concluded that their findings are in line with the hypothesis that an abnormally shaped, peg, or aplasia of lateral incisor and palatally displaced canine are biologically covariables in a complex of genetically related dental disturbances.

Bass $^{(17)}$ and Saiar et $\mathrm{al}^{(18)}$; found that canine malposition or impaction most frequently occurs in sufficient space and regular shape of dental arch. Bass added that only 10 patients of his experimental group had crowding with buccally impacted canines and thirty six percent of patients with impacted canines had regular arch. This result corresponds to the findings of Jacoby ${ }^{(11)}$ who reported that canine impaction can occur even if a sufficient space is present in the dental arch. Nagpal et al; ${ }^{(19)}$; found that in a sample of palatally impacted canines, the mesiodistal diameter of maxillary incisors crown was less than that of the control group. Also, Carvalho et $\mathrm{al}^{(20)}$ in their work concluded that the appearance of small sized permanent incisors in initial permanent dentition may be an indicator of future impaction of the canines, especially if it is combined with other dental anomalies. However, Jacoby found crowding in $15 \%$ of palatally impacted canine cases.

Sajnani and $\mathrm{King}^{(21)}$ concluded that both buccally and palatally impacted canines are associated with other odontogenic anomalies including hypodontia, enamel hypoplasia, transposition of other teeth and supernumerary teeth.

On the other hand, Brenchley and Oliver ${ }^{(22)}$ are not in line with the relation between canine impaction and an anomaly of maxillary lateral incisor. They did not have a control group as the other works. Their control group was the maxillary lateral incisors on other side of impacted canines. 
Egyptian

Orthodontic Journal

The work done by Purnell et al; ${ }^{(23)}$ goes in line with the results found by this work, in that they concluded that in patients with impacted canines(either palatally or labially), the roots of lateral incisors are angulated mesially or distally in relation to patients with normally erupted canines.

These results were in agreement with the study done by Kanavakis et al; ${ }^{(24)}$ who found that the roots of lateral incisors adjacent to palatally impacted canines were more angulated in comparison to lateral incisors adjacent to canines without impaction with the use of panoramic radiographs. They added that early diagnosis may prevent future canine palatal impaction by visual observation of crown root angulation of adjacent lateral incisor.

Panoramic radiographs have been used for many years as routine radiographs in orthodontic treatment and to diagnose impacted canines. However, in this study, CBCT was used as a primary mean for diagnosis because, panoramic radiograph was unreliable for determining the position of impacted canine in relation to adjacent lateral incisor and resorption of adjacent teeth as described by Ericson and Kurol, 1987 ${ }^{(25)}$.

Kanavakis et al; ${ }^{(24)}$ proposed that panoramic radiographs tend to overestimate mesial angulation of lateral incisor in comparison to CBCT. They stated that bucco-lingual tooth angulation may affect mesio-distal measurements on panoramic radiographs. So, an error in using two dimensional image during predicting three dimensional structures. However, we found no significant difference between $\mathrm{CBCT}$ and panorama.

Shen et al; ${ }^{(26)}$ and Knosel et al;(27) studied crown root angulation of maxillary central incisor from another point of view. They determined crown root angulation to indicate this angle difference with different types of malocclusion using cephalometric radiographs. They concluded that the class II division 2 malocclusion group had a significantly greater crown root angulation. McIntyre et al; ${ }^{(28)}$ added that shorter root, longer crown and axial bending of central incisor can cause deep bite in class II division 2 malocclusion leading to limit the amount of palatal root torque in fixed appliance treatment. 
Egyptian

Orthodontic Journal

\section{CONCLUSION}

1- The root of maxillary lateral incisor adjacent to palatally or labially impacted canines are more angulated than that adjacent to normally erupted canines.

2- Orthodontist can predict impaction of the canine by examination of crown-root angulation of the lateral incisors.

3- Since insignificant difference was found between panorama and CBCT in determining crown-root angulation, panoramic radiography is sufficient to investigate this angle to reduce un-needed radiation exposure of CBCT unless other causes are in need for CBCT to complete the diagnosis.

\section{REFERENCES}

1- Al-Ansari NB, Ghaib NH, Al-Naimi SH. Diagnosis and localization of the maxillary impacted canines by using dental multi-slice computed tomography $3 \mathrm{D}$ view and reconstructed panoramic $2 \mathrm{D}$ view. J Bagh College Dentistry.2014; 26(1).

2- Uday NM, Kamath P, Kumar V, Scindhia R, RaghurajMB, Rozario J. Comparison of axial and sagittal views for angulation, cusp tip distance and alveolus width in maxillary impacted canines using CBCT. jorthodr2014; 2(1):21-6.

3- Ericson S, Kurol J. Early treatment of palatally erupting maxillary canines by extraction of the primary canines. Eur J Orthod. 1988; 94: 503-13.

4- Kim Y, Hyun H, Jang K. The position of maxillary canine impactions and the influenced factors to adjacent root resorption in the Korean population. Eur J Orthod. 2011;2(8):5-24.

5- Coulter J, Richardson A. Normal eruption of the maxillary canine quantified in three dimensions. . Eur J Orthod. 1997; 19:171-83. 
Egyptian

Orthodontic Journal

6- Alqerban A, Jacobs R, Fieuws S and Willems G. Comparison of two cone beam computed tomographic systems versus panoramic imaging for localization of impacted maxillary canines and detection of root resorption. Eur J Orthod. 2011; 33: 93-102.

7- Mercuri E, Cassetta M, Cavallini C, Vicari D, Leonardi R, Barbato E. Dental anomalies and clinical features in patients with maxillary canine impaction. Angle Orthod.2013; 83(1):22-8.

8- Peck S, Peck L, Kataja M. Prevalance of tooth agenesis and peg-shaped maxillary lateral incisor associated with palatally displaced canine (PDC) anomaly. Am J Orthod Dentofacial Orthop. 1996;110(4):441-3.

9- Sacerdoti R and Baccetti T. Dentoskeletal features associated with unilateral or bilateral palatal displacement of maxillary canines. Angle Orthod.2004; 74(6):725-32.

10- Becker A, Sharabi S, Chaushu S. Maxillary tooth size variation in dentitions with palatal canine displacement. Eur J Orthod. 2002; 24 (3):313-8.

11- Jacoby H. The etiology of maxillary canine impactions. Am J Orthod Dentofacial Orthop.1983; 84 (2):125-32.

12- Becker A. Palatal canine displacement: guidance theory or an anomaly of genetic origin? Angle Orthod.2004; 65(2):95-102.

13- Peck S, Peck L, Kataja M. The palatally displaced canine as a dental anomaly of genetic origin. Angle Orthod.1994; 64(4):249-56.

14- Peck S, Peck L, Kataja M. Concomitant occurrence of canine malposition and tooth agenesis: evidence of orofacial genetic fields. Am J Orthod Dentofacial Orthop. 2002; 122(6) :657-60.

15- Mossey PA, Campbell HM, Luffingham JK. The palatal canine and the adjacent lateral incisor: a study of west of Scotland population. Br J Orthod. 1994; 21:169-74. 
Egyptian

Orthodontic Journal

16- Liuk IW, Olive RJ, Griffin M, Monsour P. Maxillary lateral incisor morphology and palatally displaced canines: a case controlled conebeam volumetric tomography study. Am J Orthod Dentofacial Orthop.2013; 143(4):522-6.

17- Bass TB. Observation on the misplaced upper canine tooth. Dent Pract Dent Rec.1967;18(1):25-33.

18- Saiar M, Rebellato J, Sheats RD. Palatal displacement of canines and maxillary skeletal width. Am J Orthod Dentofacial Orthop.2006; 129(4) :511-9.

19- Nagpal A, Pai KM, Sharma G. Palatal and labially impacted maxillary canine associated dental anomalies: a comparative study. J Contemp Dent Pract. 2009; 10(4):67-74.

20- Carvallo AB, Motta RH, Carvallo EM. Relation between agenesis and shape anomally of maxillary lateral incisors and canine impaction. Dental Press J Orthod. 2012; 17(6) Maringa Nov./Dec.

21- Sajnani A, king N. Dental anomalies associated with buccally and palatally-impacted maxillary canines. Journal of Investigative and Clinical Dentistry. 2014; 5,208-13.

22- Brenchley Z, Oliver RG. Morphology of anterior teeth associated with displaced canines. Br J Orthod.1997; 24(1):41-5.

23- Purnell SP, Kau C, Javed A, Souccar N, Vbachos C. Investigation of lateral incisor crown root angulation and impacted maxillary canines. 2014; MAI 52/06(E).

24- Kanavakis G, Curran KM, Wiseman KC, Barone N, Finkeiman M, Srinivasan S, Lee M, Trotman C. Evaluation of crown-root angulation of lateral incisors adjacent to palatally impacted canines. Progress in Orthodontics. 2015; 16:4.

25- Ericson S, Kurol J. Radiographic examination of ectopically erupting maxillary canines. Am J Orthod Dentofacial Orthop.1987;91(6) :483-92. 
Egyptian

Orthodontic Journal

26- Shen YW, Hsu JT,Wang YH, Huang HL, Fuh LJ. The collum angle of the maxillary central incisors in patients with different types of malocclusion. Journal of Dental Science. 2012; 7, 72-6.

27- Knosel M, Jung K, Attin T, Engelke W, Meesenburg D, Rudolph, LG, Attin R. On the interaction between incisor crown root morphology and third order angulation. Angle Orthod.2009; 79:454-61.

28- Mclntyre G, Millett DT. Crown-root shape of the permanent maxillary central incisor. Angle Orthod.2003; 73: 710-15. 